\title{
Prevalência de Disfunção Tireoidiana e Anticorpos Antitireoidianos em Pacientes Portadores de Diabetes Melito Tipo 1 e em seus Familiares de Primeiro Grau
}

\begin{abstract}
RESUMO
O diabetes melito tipo 1 (DM1) freqüentemente encontra-se associado à doença auto-imune da tireóide (DAT). A prevalência de DAT varia de $3 \%$ a $50 \%$ entre diabéticos, e é maior também entre seus familiares, comparada à população geral. Objetivos: Investigar a prevalência da DAT em pacientes com DM1, avaliar possíveis diferenças de comportamento clínico-evolutivo do DM1 entre diabéticos com e sem DAT e estudar a prevalência de DAT nos familiares dos pacientes diabéticos. Materiais e Métodos: Os prontuários de 124 diabéticos tipo 1 foram revisados e coletados os dados referentes à função e aos anticorpos tireoidianos; pacientes com e sem DAT foram comparados em relação à média de $\mathrm{Hb}$ glicosilada, complicações agudas e crônicas, idade ao diagnóstico e tempo de evolução do DM, dose de insulina e outros. Um estudo caso-controle foi realizado com 54 familiares em primeiro grau destes pacientes; foram avaliadas a função tireoidiana e a presença de anticorpos antitireoidianos em 32 familiares de diabéticos sem DAT e 22 familiares de diabéticos com DAT. Resultados: As prevalências de DAT e de disfunção hormonal entre os diabéticos foram de $35,5 \%$ e $19,3 \%$, respectivamente. Quanto à avaliação dos parâmetros de evolução do DM1, comportamento clínico e controle metabólico não houve diferenças significantes entre os diabéticos com e sem DAT. Houve maior prevalência de DAT nos familiares de diabéticos com DAT do que no grupo dos familiares dos diabéticos sem DAT, sem diferença significativa quanto à prevalência de disfunção hormonal. Conclusões: A prevalência de doença auto-imune de tireóide em diabéticos e em seus familiares é elevada, justificando-se, nesses casos, a investigação rotineira da função tireoidiana, particularmente dos familiares de primeiro grau de diabéticos com DAT. (Arq Bras Endocrinol Metab 2008; 52/6:985-993)
\end{abstract}

Descritores: Diabetes melito tipo 1; Doença auto-imune da tireóide; Anticorpos antitireoidianos; Familiares

\begin{abstract}
Prevalence of Thyroid Dysfunction and Antithyroid Antibodies in Type 1 Diabetic Mellitus Patients and their First Degree Relatives.

Diabetes Mellius Type 1 (DM1) is frequently associated to Autoimmune Thyroid Disease (AITD). The prevalence of AITD among diabetic patients varies between 3 to $50 \%$ and the incidence is also big among their family members, when compared to the population in general. Objectives: To investigate the prevalence of AITD in patients with DM1; to evaluate possible differences concerning the clinical-evolutive behavior of DM1 among diabetic patients with or without AITD and to study the prevalence of AITD among the diabetes patients' relatives. Materials and Methods: 124 prontuaries of diabetic patients (type 1) were revised and data was gathered concerning the thyroid function and the anti-thyroid antibodies. Patients with and without AITD were compared in relation to the level of glycosylated hemoglobin, the presence of acute and chronic complications, the age of the patient at the time of the
\end{abstract}

original

\author{
RICARDO RODRIGUES \\ FABRÍCIA TORRES GONÇALVES \\ Paulo Tannus Jorge
}

Serviço de Endocrinologia e Metabologia do Hospital

de Clínicas da UFU,

Uberlândia, MG, Brasil. 
diagnosis, time of evolution of the disease, daily dose of insulin and other factors. A control case study was conducted with 54 first degree relatives of the diabetic patients who took part in the study; the thyroid function as well as the presence of anti-thyroid antibodies were evaluated in 32 of those first degree relatives with AITD, and in 22 of those without AITD. Results: The prevalence of AITD and of hormonal dysfunction among diabetic patients was $35.5 \%$ and $19.3 \%$, respectively. No significant differences were found between groups in respect to clinical outcome or to diabetic chronic complications. However, prevalence of AITD and hormonal dysfunction were found to be higher among first degree relatives of diabetic patients with AITD than among relatives of diabetic patients without AITD. Conclusions: The prevalence of autoimmune thyroid disease in diabetic patients and in their first degree relatives is high. Thyroid function screening is therefore justified in these cases, especially in first degree relatives of diabetics with autoimmune thyroid disease. (Arq Bras Endocrinol Metab 2008; 52/6:985-993)

Keywords: Type 1 diabetes mellitus; Thyroid autoimmune disease; Antithyroid antibodies; Relatives

\section{INTRODUÇÃO}

As siat S PRIMEIRAS EVIDÊNCIAS da natureza auto-imune do diabetes melito tipo $1(\mathrm{DMl})$ vieram da observação de esta doença estar freqüentemente associada a outras endocrinopatias auto-imunes, como tireoidite de Hashimoto, doença de Graves, adrenalite, insuficiência ovariana primária, anemia perniciosa e doença celíaca (1). A identificação de auto-anticorpos dirigidos contra componentes da célula- $\beta$, como anticorpos antiinsulina, anti-GAD (descarboxilase do ácido glutâmico) e antiilhotas, reforçaram a importância da auto-imunidade na etiologia desta doença. No entanto, não obstante esse claro componente imunológico, é uma doença heterogênea com diversas formas e graus de processo imune envolvidos e com suscetibilidade genética, como a base para o seu desenvolver, estando sua etiopatogenia, ainda, com diversos pontos a serem esclarecidos, uma vez que fatores ambientais parecem ter importante papel em sua gênese $(2,3)$.

As semelhanças no padrão genético de herança e suscetibilidade, além do mecanismo auto-imune compartilhado entre essas endocrinopatias, associadas ao aumento na incidência delas em todo o mundo, têm estimulado pesquisadores a estudar, epidemiologicamente, não só os portadores destas patologias como seus familiares de primeiro grau (4-7). Estudos mundiais revelam diferentes valores de prevalência de anticorpos antitireoidianos em pacientes com DMl, que variam de $3 \%$ a $50 \%(8)$.

As diferenças no comportamento do diabetes entre pacientes portadores e não-portadores de tireoidopatia auto-imune também têm sido objeto de estudos, com alguns autores encontrando diabetes mais grave entre pacientes que haviam desenvolvido auto-imunidade contra a tireóide $(9,10)$, embora outros não tenham encontrado essas diferenças (7,11-15). No Brasil, essa mesma divergência na prevalência de DAT no DMl tem-se reproduzido nos poucos estudos realizados, com alguns autores encontrando números que variam de $9 \%$ a 33\% (16-19). Novos estudos epidemiológicos contribuiriam para o conhecimento da real prevalência da DAT entre os DMl e em seus familiares e também para a comprovação de influências da associação entre as doenças auto-imunes sobre o curso de cada doença.

O presente trabalho tem por objetivo determinar, na população estudada, a prevalência de DAT e a disfunção tireoidiana em pacientes portadores de DMl e em seus familiares de primeiro grau, avaliar possíveis diferenças de comportamento clínico-evolutivo do DMl entre os diabéticos portadores ou não de DAT e, além disso, avaliar se existe diferença na prevalência de DAT entre familiares de diabéticos tipo 1 portadores e familiares de diabéticos não-portadores de DAT.

\section{MÉTODOS}

A primeira fase tratou de estudo retrospectivo que avaliou os prontuários dos pacientes portadores de DMl, que se encontravam em acompanhamento ao longo do último ano nos serviços de endocrinologia do Hospital de Clínicas da Faculdade de Medicina da Universidade Federal de Uberlândia (UFU), e em ambulatório de 
referência para jovens diabéticos da Secretaria Municipal de Saúde de Uberlândia. Foram incluídos os pacientes com diagnóstico de DMl, conforme a classificação adotada pela Associação Americana de Diabetes (ADA) (20), caracterizados pela dependência de insulina desde o diagnóstico associado às características clínicas e epidemiológicas clássicas do início do DMl (poliúria, polidipsia, emagrecimento, tendência à cetoacidose e faixa etária de início dos sintomas entre a infância e a adolescência) ou ainda pacientes insulinodependentes com dosagem de anti-GAD positiva. Nos casos em que a classificação não estivesse clara, como em suspeitos de maturity onset diabetes of young (MODY) ou diabetes gestacional, os pacientes foram excluídos.

Todas as dosagens de anticorpos antitireoperoxidase (anti-TPO), antitireoglobulina (ATG) e tireotrofina (TSH) foram realizadas por quimioluminescência (Immulite-Diagnostic Products Corporation), utilizandose doseamento imunométrico seqüencial. A DAT foi definida pela presença de anticorpos antitireoidianos reagentes acima do limite da normalidade $(35 \mathrm{IU} / \mathrm{ml}$ para o anti-TPO e até $40 \mathrm{IU} / \mathrm{ml}$ para o ATG). A disfunção tireoidiana foi considerada quando o nível de TSH era superior a 4,2 $\mathrm{mIU} / \mathrm{ml}$, no caso de hipotireoidismo, e menor que $0,3 \mathrm{mIU} / \mathrm{ml}$, no caso de hipertireoidismo.

Os dados laboratoriais utilizados para avaliar o controle metabólico do diabetes foram os níveis de hemoglobina glicosilada $(\mathrm{HbAlc})$, medidos por meio de imunoturbidimetria, e, portanto, considerada como valor limítrofe de normalidade $4,5 \%$ a $5,2 \%$ (21). A freqüência de complicações agudas, como episódios de cetoacidose diabética e hipoglicemias graves ao longo do último ano, e a presença de complicações crônicas, avaliadas pela presença de retinopatia diabética em fundoscopia, nefropatia diabética na presença de micro ou macroalbuminúrias e neuropatia diabética por meio de exame clínico descrito em prontuário, foram as outras variáveis estudadas relacionadas com o controle metabólico.

Para a avaliação de possíveis diferenças no comportamento clínico do diabetes nos dois grupos, também foram considerados outros parâmetros, como a dose utilizada de insulina por quilograma $(\mathrm{kg})$ de peso por dia, o tempo de evolução e a idade de início do diabetes, bem como a presença de outras patologias autoimunes associadas.

A segunda fase do estudo, caracterizada por ser do tipo caso-controle, foi realizada com os familiares de primeiro grau desses pacientes portadores de DMl, subdivididos em dois grupos: um grupo de familiares de pacientes diabéticos tipo 1 sem DAT e outro grupo de familiares de diabéticos tipo 1 com DAT. Em ambos foram realizadas dosagens TSH e dos anticorpos antitireoidianos, por quimioluminescência. A classificação quanto ao status da função tireoidiana dos familiares dos diabéticos seguiu o mesmo padrão utilizado para a primeira fase do estudo, conforme já descrito.

Todos os familiares dos pacientes diabéticos tipo 1 acompanhados no serviço de endocrinologia foram convidados a participar do estudo e aqueles que aceitaram assinaram o termo de consentimento esclarecido. O projeto foi analisado e aprovado pelo Comitê de Ética em Pesquisa (CEP) da UFU.

\section{Análise estatística}

Para as análises estatísticas, as variáveis foram testadas quanto a sua normalidade pelo teste de KolmogorovSmirnov. Os resultados foram apresentados com medidas de posição e dispersão, como média e desvio-padrão para as variáveis com distribuição normal. Foi utilizado o teste $t$ de Student para comparação entre duas médias e o teste de comparação de proporções (teste z) (22). Todos os testes foram aplicados utilizando-se intervalo de confiança de $95 \%$ e considerados estatisticamente significativos quando $\mathrm{p}<0,05$.

\section{RESULTADOS}

$\mathrm{Na}$ primeira fase do estudo, foram avaliados os $124 \mathrm{pa}-$ cientes portadores de DMl que se encontravam em acompanhamento ativo nos serviços referidos, sendo 64 $(51,6 \%)$ do sexo masculino e $60(48,4 \%)$ do sexo feminino. A avaliação da função tireoidiana e da DAT, caracterizada pela presença de anticorpos antitireoidianos, desse grupo de pessoas mostrou algum grau de doença tireoidiana em 49 pacientes $(39,5 \%)$, visto que $44(35,5 \%)$ apresentavam anticorpos positivos contra a tireóide e cinco $(4 \%)$ eram portadores de hipotireoidismo primário com anticorpos negativos. As características clínico-demográficas dos pacientes diabéticos em geral, com DAT e sem DAT estão descritas na Tabela 1.

A prevalência dos dois tipos de anticorpos antitireoidianos entre os diabéticos em geral foi elevada, sendo o anti-TPO mais freqüentemente encontrado $(31 \%)$ que o anti- $\operatorname{Tg}(16 \%)(\mathrm{p}=0,002)$. Entre as 60 pacientes do sexo feminino e os 64 do grupo masculino, 25 $(41,4 \%)$ e $19(29,7 \%)$, respectivamente, apresentavam DAT $(\mathrm{p}=0,08)$. 
Nos pacientes com DAT, a disfunção tireoidiana associada foi encontrada em 24 (54,5\%); e, embora tenha sido mais freqüente entre as mulheres $(26,6 \%)$ do que entre os homens $(12,9 \%)$, não foi estatisticamente significante $(\mathrm{p}=0,1)$. $\mathrm{O}$ hipotireoidismo por tireoidite de Hashimoto estava presente em 22 (91,7\%) destes pacientes e o hipertireoidismo por doença de Graves em dois $(8,3 \%)$. Os níveis médios de TSH dos pacientes diabéticos com DAT foram superiores em relação ao outro grupo $(\mathrm{p}=0,02)$, como demonstrado na Tabela 1.

O tempo de evolução da doença tireoidiana no grupo de pacientes diabéticos que apresentava DAT foi, em média, de 2,32 anos ( $\mathrm{DP}=3,05)$. O diagnóstico de DAT foi prévio ao do diabetes $(4,1 \%)$, concomitante $(29,2 \%)$ ou posterior $(66,7 \%)(\mathrm{p}=0,0001)$.

Tabela 1. Características clínicas dos pacientes diabéticos com e sem doença tireoidiana.

\begin{tabular}{|c|c|c|c|c|}
\hline \multirow{2}{*}{$\begin{array}{l}\text { Parâmetros } \\
\text { avaliados }\end{array}$} & \multicolumn{3}{|c|}{ Pacientes avaliados } & \\
\hline & Geral & Com DAT & Sem DAT & \\
\hline $\begin{array}{l}\text { Número de } \\
\text { pacientes }\end{array}$ & $124^{\circ}$ & 44 & 75 & \\
\hline $\begin{array}{l}\text { Idade } \\
\text { (anos* e DP) }\end{array}$ & $16,7(6,3)$ & $16,6(5,6)$ & $17,0(6,7)$ & $\mathrm{p}=0,67^{* *}$ \\
\hline $\begin{array}{l}\mathrm{Hb} \text { glicosilada* } \\
\text { (\%) }\end{array}$ & 10,3 & 10,1 & 10,5 & $\mathrm{p}=0,37^{* *}$ \\
\hline $\begin{array}{l}\text { Dose de } \\
\text { insulina* } \\
\text { (UI/kg/dia) }\end{array}$ & 0,83 & 0,82 & 0,83 & $\mathrm{P}=0,42^{* *}$ \\
\hline \multicolumn{5}{|l|}{ Episódios } \\
\hline $\begin{array}{l}\text { Complicações } \\
\text { agudas\& } \%)\end{array}$ & 21 & 20,4 & 20,0 & $\mathrm{p}=0,49^{* * *}$ \\
\hline $\begin{array}{l}\text { Complicações } \\
\text { crônicas (\%) }\end{array}$ & 16,1 & 18,2 & 13,3 & $\mathrm{p}=0,25^{\star * *}$ \\
\hline $\begin{array}{l}\text { Duração do } \\
\text { DM* (em anos) }^{*}\end{array}$ & 6,3 & 5,4 & 6,7 & $\mathrm{p}=0,10^{* *}$ \\
\hline $\mathrm{IMC}^{*}\left(\mathrm{~kg} / \mathrm{m}^{2}\right)$ & 19,8 & 20,7 & 19,3 & $\mathrm{P}=0,02^{* *}$ \\
\hline $\begin{array}{l}\text { Idade ao } \\
\text { diagnóstico do } \\
\mathrm{DM}^{*}(\mathrm{DP})\end{array}$ & $10,4(6,3)$ & $11,6(5,8)$ & $9,8(6,3)$ & $p=0,06^{* *}$ \\
\hline $\begin{array}{l}\text { Níveis médios } \\
\text { de TSH } \\
(\text { microUl/ml) }\end{array}$ & 3,6 & 12,1 & 2,4 & $\mathrm{p}=0,02^{* *}$ \\
\hline \multicolumn{5}{|c|}{ 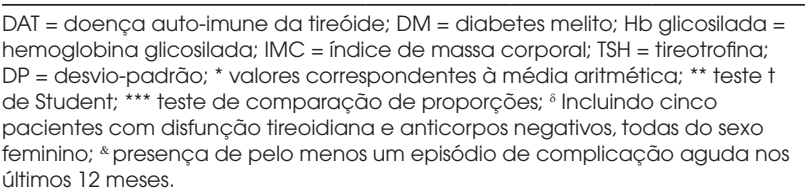 } \\
\hline
\end{tabular}

$\mathrm{Na}$ avaliação dos parâmetros clínicos e laboratoriais dos grupos de pacientes diabéticos tipo l, com e sem DAT, observou-se diferença estatisticamente significante apenas em relação ao $\operatorname{IMC}(\mathrm{p}=0,02)$ (Tabela 1$)$.

Conforme os dados da Tabela 2, a média de idade, o IMC e o tempo médio de evolução do diabetes foram semelhantes entre os pacientes diabéticos tipo 1 apenas com anticorpos positivos comparados àqueles que também apresentavam disfunção hormonal. Ainda que níveis maiores de dose diária de insulina, média de hemoglobina glicosilada e prevalência de complicações crônicas tenham sido encontrados entre aqueles que apresentavam DAT com alteração dos níveis hormonais, estas diferenças não foram estatisticamente significantes, exceto em relação ao tempo de diagnóstico de $\operatorname{DAT}(\mathrm{p}=0,04)$.

Tabela 2. Avaliação de parâmetros clínicos e laboratoriais de pacientes diabéticos tipo 1 portadores de DAT, comparados quanto à presença ou não de disfunção hormonal tireoidiana.

\begin{tabular}{|c|c|c|c|}
\hline Parâmetros & $\begin{array}{l}\text { DAT com } \\
\text { disfunção } \\
\text { tireoidiana }\end{array}$ & $\begin{array}{l}\text { DAT sem } \\
\text { disfunção } \\
\text { tireoidiana }\end{array}$ & \\
\hline $\begin{array}{l}\text { Número de } \\
\text { pacientes }\end{array}$ & 24 & 20 & \\
\hline Idade* (anos) (DP) & $17,6(5,1)$ & $16,3(6,3)$ & $p=0,48^{* *}$ \\
\hline $\begin{array}{l}\text { Tempo de diabetes* } \\
\text { (anos) }\end{array}$ & 6 & 4,7 & $\mathrm{p}=0,41^{* *}$ \\
\hline $\mathrm{IMC}^{*}\left(\mathrm{~kg} / \mathrm{m}^{2}\right)$ & 21,2 & 20,1 & $p=0,45^{* *}$ \\
\hline $\begin{array}{l}\text { Hemoglobina } \\
\text { glicosilada* (\%) }\end{array}$ & 10,5 & 9,6 & $p=0,32^{* *}$ \\
\hline $\begin{array}{l}\text { Dose de insulina* } \\
\text { (Ul/kg/dia) }\end{array}$ & 0,91 & 0,71 & $p=0,09^{* *}$ \\
\hline $\begin{array}{l}\text { Presença de } \\
\text { complicações } \\
\text { crônicas do } \\
\text { diabetes (\%) }\end{array}$ & 25 & 10 & $\mathrm{P}=0,08^{* * *}$ \\
\hline $\begin{array}{l}\text { Episódios de } \\
\text { complicações } \\
\text { agudas do } \\
\left.\text { diabetes\& }^{2} \%\right)\end{array}$ & 16,7 & 25 & $\mathrm{p}=0,22^{* * *}$ \\
\hline $\begin{array}{l}\text { Tempo de } \\
\text { diagnóstico da DAT* } \\
\text { (anos) }\end{array}$ & 3,1 & 1,3 & $p=0,04^{* *}$ \\
\hline
\end{tabular}

* valores correspondentes à média; ${ }^{* *}$ teste t de Student; *** teste z; DAT = doença auto-imune da tireóide; $I M C$ = índice de massa corporal; $F=$ sexo feminino; $M$ = sexo masculino; DP = desvio-padrão; \& presença de pelo menos um episódio de complicação aguda nos últimos 12 meses. 
$\mathrm{Na}$ avaliação dos pacientes diabéticos em relação à faixa etária, estes foram subdivididos em três grupos, quais sejam, até 10 anos de idade, de 11 a 15 anos e maiores de 15 anos. A prevalência de DAT foi de $26 \%$, $28,6 \%$ e $34,8 \%$, respectivamente, e a presença de disfunção hormonal foi encontrada em 13,1\%, 25,7\% e $25,7 \%$, respectivamente, sem diferença estatística significante entre estes três grupos de faixa etária. De todas as outras variáveis analisadas ( $\mathrm{Hb}$ glicosilada, dose de insulina, complicações agudas e crônicas) apenas a maior prevalência de complicações crônicas no grupo de faixa etária acima de 15 anos foi estatisticamente significante $(\mathrm{p}=0,04)$ (Tabela 3$)$.

A segunda fase do estudo avaliou a prevalência de DAT entre familiares de pacientes diabéticos que não apresentavam DAT $(\mathrm{n}=32)$ e familiares de pacientes diabéticos com DAT $(n=22)$. As Tabelas 4 e 5 mostram as características epidemiológicas dos familiares em geral.

Observou-se prevalência de DAT de 21,9\% no grupo de familiares dos que não tinham doença tireoidiana associada, e de $63,6 \%$ no grupo de familiares de portadores de DAT $(\mathrm{p}=0,005)$. $\mathrm{O}$ valor de odds ratio (OR) encontrado indicou maior risco de desenvolvimento de DAT entre familiares de pacientes diabéticos tipo 1 com DAT que entre familiares dos diabéticos tipo 1 sem DAT $(\mathrm{OR}=4,0 ; \mathrm{IC}=95 \%)$. Conforme apresentado no Gráfico 1, a prevalência de ambos os

Tabela 3. Avaliação dos parâmetros clínicos e laboratoriais entre diabéticos do tipo 1, conforme a idade dos pacientes.

\begin{tabular}{lccccc}
\hline Parâmetros & $\begin{array}{c}\leq 10 \\
\text { anos }\end{array}$ & $\begin{array}{c}11 \text { a } 15 \\
\text { anos }\end{array}$ & $\begin{array}{c}>15 \\
\text { anos }\end{array}$ & \\
$\begin{array}{l}\text { Prevalência de DAT (\%) } \\
26\end{array}$ & 28,6 & 34,8 & $\mathrm{p}=0,67^{* *}$ \\
$\begin{array}{l}\text { Prevalência de } \\
\text { disfunção hormonal } \\
\text { tireoidiana (\%) }\end{array}$ & 13,1 & 25,7 & 25,7 & $\mathrm{p}=0,43^{* *}$ \\
$\begin{array}{l}\text { Hemoglobina } \\
\text { glicosilada* (\%) }\end{array}$ & 8,9 & 10,7 & 10,7 & $\mathrm{p}=0,97^{* * *}$ \\
$\begin{array}{l}\text { Dose de insulina* } \\
\text { (Ul/kg/dia) }\end{array}$ & 0,8 & 0,9 & 0,8 & $\mathrm{p}=0,35^{* * *}$ \\
$\begin{array}{l}\text { Prevalência de } \\
\text { episódio de } \\
\text { complicações } \\
\text { agudas (\%) }\end{array}$ & 13,1 & 31,5 & 16,7 & $\mathrm{p}=0,13^{* *}$ \\
$\begin{array}{l}\text { Prevalência de } \\
\text { complicações } \\
\text { crônicas (\%) }\end{array}$ & 8,7 & 2,8 & 25,7 & $\mathrm{p}=0,04^{* *}$ \\
\hline
\end{tabular}

DAT = doença auto-imune da tireóide; * valores correspondentes à média; ** teste $x^{2} ; * * *$ teste † de Student; \& presença de pelo menos um episódio de complicação aguda nos últimos 12 meses.
Tabela 4. Características dos familiares dos pacientes diabéticos estudados.

\begin{tabular}{|c|c|}
\hline Número de familiares & 54 \\
\hline Distribuição quanto ao sexo (\%) & $\mathrm{F}: 55,5 / \mathrm{M}: 44,5$ \\
\hline Idade* (anos) (DP) & $34,4(15,7)$ \\
\hline Prevalência - DAT (\%) & 38 \\
\hline Prevalência - anti-TPO (\%) & 33,3 \\
\hline Prevalência - ATG (\%) & 29,6 \\
\hline Prevalência de disfunção hormonal (\%) & 18,5 \\
\hline Prevalência de hipotireoidismo (\%) & 14,8 \\
\hline Prevalência de hipertireoidismo (\%) & 3,7 \\
\hline
\end{tabular}

* valor correspondente à média aritmética; DAT = doença auto-imune da tireóide; $F=$ sexo feminino; $M$ = sexo masculino; $D P=$ desvio-padrão.

anticorpos, anti-TPO e ATG, foi elevada entre os familiares dos pacientes diabéticos com associação de DAT (50\% e 54\%, respectivamente) comparada à encontrada nos familiares daqueles pacientes que não apresentavam esta associação. Neste último grupo, o discreto predomínio de positividade do anti-TPO (22\% versus 13\%) em relação ao ATG não teve relevância estatística $(\mathrm{p}=0,14)$.

Gráfico 1. Prevalência de anticorpos antitireoidianos (antiTPO e ATG) e DAT entre familiares dos diabéticos tipo 1 com e sem DAT associada.

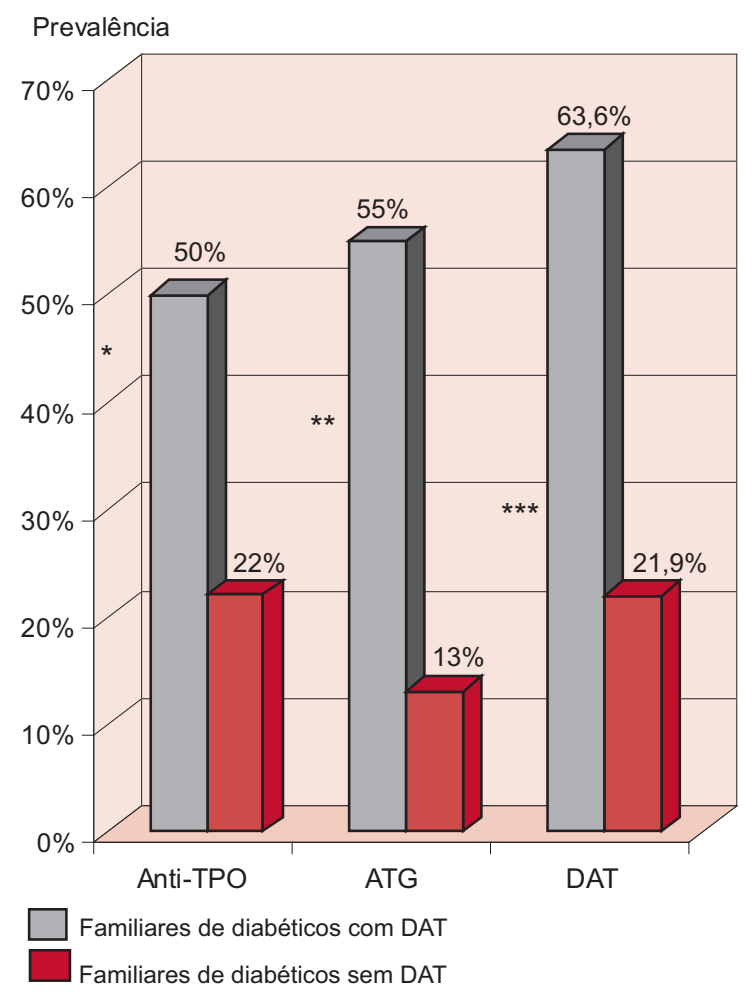


Tabela 5. Prevalência da DAT e disfunção hormonal tireoidiana entre mães, pais, irmãs e irmãos no grupo de familiares dos pacientes diabéticos com DAT e no grupo de familiares dos pacientes diabéticos sem DAT.

\begin{tabular}{|c|c|c|c|c|c|c|c|c|}
\hline & \multicolumn{4}{|c|}{ Familiares do grupo de pacientes diabéticos com DAT } & \multicolumn{4}{|c|}{ Familiares do grupo de pacientes diabéticos sem DAT } \\
\hline & $n$ & $\begin{array}{l}\text { Média de } \\
\text { idade }\end{array}$ & $\begin{array}{c}\text { Prevalência } \\
\text { DAT (\%) }\end{array}$ & $\begin{array}{c}\text { Disfunção } \\
\text { hormonal (\%) }\end{array}$ & $\mathrm{n}$ & $\begin{array}{l}\text { Média de } \\
\text { idade }\end{array}$ & $\begin{array}{c}\text { Prevalência } \\
\text { DAT (\%) }\end{array}$ & $\begin{array}{c}\text { Disfunção } \\
\text { hormonal (\%) }\end{array}$ \\
\hline Geral & 22 & 28,6 & 63,6 & 27,3 & 32 & 38,4 & 21,9 & 12,5 \\
\hline Mães & 7 & 41,1 & 71,4 & 42,8 & 14 & 44,9 & 42,8 & 21,4 \\
\hline Pais & 4 & 43,7 & 75 & 50 & 8 & 50,1 & 12,5 & 12,5 \\
\hline Irmãs & 6 & 13,3 & 83,3 & 16,7 & 3 & 17,3 & 0 & 0 \\
\hline Irmãos & 5 & 17 & 20 & 0 & 7 & 19,4 & 0 & 0 \\
\hline
\end{tabular}

DAT = doença auto-imune da tireóide; $\mathrm{n}$ = número de indivíduos

DAT = doença auto-imune da tireóide; $\mathrm{ATG}=$ anticorpo antitireoglobulina; anti-TPO = anticorpo antitireoperoxidase; ${ }^{*} \mathrm{P}=0,009 ;{ }^{* *} \mathrm{P}=0,002 ;{ }^{* * *} \mathrm{P}=0,005$

Quanto à disfunção hormonal, também ocorreu com maior freqüência entre os familiares dos pacientes diabéticos com DAT associada, porém a diferença encontrada não foi estatisticamente significante $(\mathrm{p}=0,06)$ (Tabela 1). Os níveis médios do TSH entre estes dois grupos foram diferentes, com níveis mais altos entre os familiares dos diabéticos com DAT associada (10,7 $\mu \mathrm{UI} / \mathrm{ml}$ versus $2,12 \mu \mathrm{UI} / \mathrm{ml}$ ), porém, sem significância estatística $(\mathrm{p}=0,2)$.

Quando comparados segundo os mesmos níveis de parentesco, não se encontrou diferença estatística significante da prevalência de DAT nem de disfunção tireoidiana entre os dois grupos de familiares, conforme demonstrado no Gráfico 2.

\section{DISCUSSÃo}

A população de diabéticos estudada caracterizou-se, epidemiologicamente, por se tratar de crianças e adultos jovens (média de idade de 16,7 anos) com diagnóstico de DMl no início do período puberal (em média aos 10,4 anos), dados estes condizentes com a descrição clássica da literatura em relação ao pico de incidência entre 10 a 14 anos, e o maior acometimento de crianças e adultos jovens por este tipo de diabetes $(23,24)$. Assim como em outros estudos epidemiológicos houve distribuição semelhante quanto ao sexo (24).

A prevalência de DAT encontrada na população de diabéticos tipo 1 estudada $(35,5 \%)$, é concordante com os achados clássicos de maior freqüência de outras doenças auto-imunes entre diabéticos tipo 1, principalmente a tireoidite de Hashimoto, deixando clara a necessidade de rastreamento para doença tireoidiana nessa população. Além disso, esse aumento da prevalência, quando comparado aos valores encontrados em estudos de duas a três décadas atrás $(9,17)$, foi observado em outros estudos atuais $(8,14,19,25)$. Embora tal achado possa ser real aumento da prevalência destas doenças, deve-se ressaltar que pode ser reflexo apenas de melhor sensibilidade dos métodos atuais na detecção dos portadores de anticorpos contra a tireóide.

No presente estudo, os pacientes diabéticos com DAT apresentaram níveis de TSH maiores que o grupo

Gráfico 2. Niveis médios de TSH entre os grupos de pacientes diabéticos com e sem DAT.

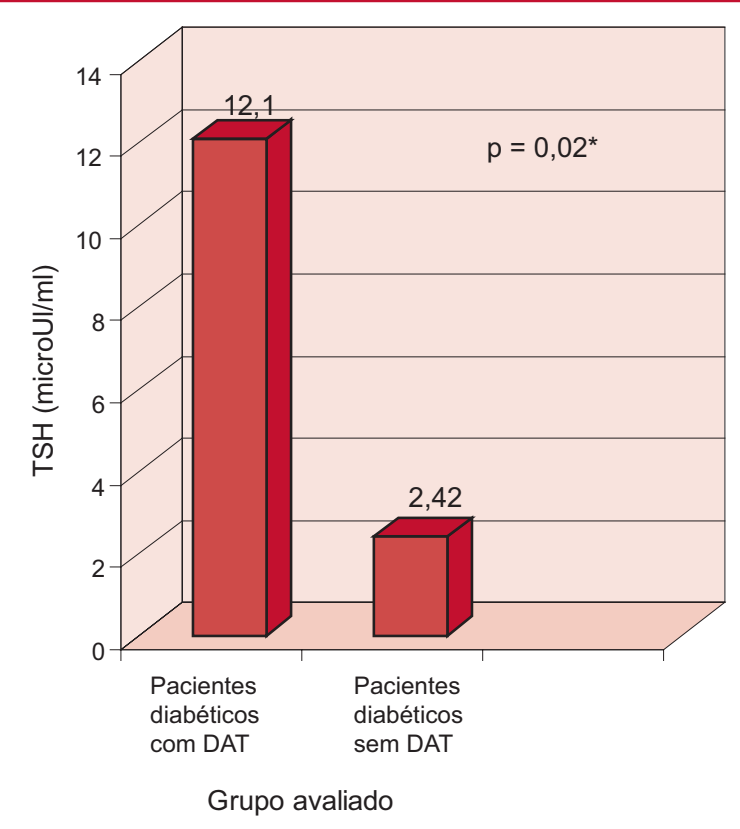

DAT = doença auto-imune da tireóide; * teste $†$ de Student. 
sem DAT, refletindo a maior freqüência de disfunção tireoidiana nesse grupo, por causa, principalmente, do hipotireoidismo. Também, a maior prevalência do anticorpo anti-TPO positivo sobre os títulos de anticorpo antitireoglobulina (ATG) observada entre os diabéticos em geral reforça a maior importância do anti-TPO na deteç̧ão da DAT já relatada em outros estudos (26).

A maior prevalência da DAT no sexo feminino, como ocorre na população geral (27), também já reportada em estudos anteriores entre diabéticos tipo 1 (25), foi tendência observada em nosso estudo. A presença de disfunção hormonal tireoidiana conforme o sexo não apresentou diferença estatística. Tal achado pode ser decorrente de maior acometimento dos diabéticos do sexo masculino quando comparados à população geral.

Entre os diabéticos portadores de DAT em nosso estudo, mais da metade apresentavam anticorpos positivos com disfunção hormonal tireoidiana, de maneira diferente do que é observado na população geral, na qual se encontra maior prevalência de DAT sem disfunção hormonal associada (28). Este fato poderia ser explicado pela hipótese de que haveria maior nível de agressividade contra a tireóide nos pacientes que possuem associação de doenças auto-imunes.

Considerando o comportamento clínico do diabetes, não se encontrou maior agressividade ou pior controle metabólico dos pacientes em decorrência da presença de DAT. Achados semelhantes foram descritos nos estudos de Molina e cols. (25) e Umpierrez e cols. (14), porém não há consenso em relação a estes dados (8,29-31). Kordonouri (8) encontrou que os diabéticos com DAT associada eram de faixa etária maior, desenvolveram diabetes em idades mais avançadas e, além disso, tinham tempo maior de evolução do diabetes. Vale ainda ressaltar que a avaliação de complicações crônicas, como retinopatia e neuropatia diabética, estão expostas a limitações intrínsecas aos estudos retrospectivos, como variabilidade interobservador e diferentes critérios de diagnóstico e classificação utilizados ao longo do tempo.

Quanto ao fato de o índice de massa corporal (IMC) ter se mostrado maior no grupo de diabéticos com DAT associada, isso provavelmente não teve implicação clínica, pois os dois grupos apresentaram IMC dentro dos limites da faixa considerada normal e não houve diferença quanto à dose de insulina diária utilizada pelos pacientes.

De maneira geral, a população de diabéticos estudada caracterizou-se pelo controle metabólico ruim, visto que a média da hemoglobina glicosilada foi de $10,34 \%$, bem acima dos alvos preconizados para o controle do diabetes, segundo a $\operatorname{ADA}(21,23,32)$. Entre as possíveis explicações para esta média elevada, foram encontrados aspectos socioeconômicos que impunham limitações ao tratamento, como incapacidade de automonitorização glicêmica, impossibilidade do uso de melhores insulinas e análogos e, por fim, de esquema de insulinoterapia intensiva, como preconiza os achados do Diabetes Control and Complications Trial (DCCT) (32). Em relação às doses de insulina, foram semelhantes aos dados da literatura, em que diabéticos com alguns anos de evolução necessitam em torno de $1,0 \mathrm{UI} / \mathrm{kg} /$ dia (33). Não obstante o mau controle metabólico, as taxas de complicações crônicas não tão elevadas, observadas nesta população estudada, muito provavelmente são decorrentes do período de evolução do diabetes relativamente curto (em média 6,3 anos). Quando se observa, especificamente, aqueles com mais de dez anos de doença tem-se elevada prevalência das complicações crônicas.

Quanto à prevalência de DAT em familiares de primeiro grau dos diabéticos tipo 1 , a avaliação laboratorial realizada na segunda fase do estudo mostrou-se maior que a prevalência na população geral encontrada na literatura que é de $2 \%$ a $10 \%$ e, além disso, foi maior entre os familiares de diabéticos que portavam DAT, do que entre os familiares de diabéticos que não eram portadores de DAT. Estes resultados são concordantes com os estudos realizados por Hanukoglu e cols. (4) e Jaeger e cols. (5), em que se encontrou maior prevalência de doenças auto-imunes órgão-específicas nos familiares de diabéticos tipo 1 .

Embora o número de familiares que compareceram para a coleta dos exames tenha sido pequeno, por causa de dificuldades para acesso ao sistema de coleta do hospital, filas prolongadas, por não residirem no mesmo município e a própria opção por não participar de tal estudo, ambos os grupos estavam expostos aos mesmos problemas. A realização desse estudo na forma de casocontrole, além de ser um dos poucos realizados, conforme extensa revisão bibliográfica, permitiu melhor avaliação da possibilidade associação de doenças autoimunes entre familiares de portadores de DMI.

Acredita-se que a realização de investigação para DAT em familiares de diabéticos tipo 1, que apresentem associação com outras doenças auto-imunes, seja procedimento importante, devendo ser realizado de maneira rotineira, visto a elevada prevalência encontrada nesta população em especial e, ainda, ao fato de que 
a doença tireoidiana precocemente diagnosticada e tratada evitaria maior morbidade para estes pacientes.

\section{CONCLUSÕES}

A prevalência de DAT em diabéticos tipo 1 em nossa população estudada é elevada em relação à população geral descrita pela literatura e bastante semelhante à prevalência de DAT encontrada em outros estudos recentes. A evolução clínica e a gravidade do DM nesses pacientes estudados não se mostraram afetadas pela presença de DAT. A presença de auto-imunidade contra a tireóide foi fator significativo para o desenvolvimento de disfunção tireoidiana. Em relação aos familiares em primeiro grau de portadores de DMl, neste estudo, concluiu-se que possuem maior prevalência de DAT e disfunção tireoidiana em relação ao descrito na literatura na população geral. A prevalência de DAT e a disfunção tireoidiana são maiores em familiares de diabéticos portadores de DAT do que em familiares de diabéticos não-portadores de DAT. Concluiu-se também pela importância do rastreamento de DAT e disfunção tireoidiana em familiares de portadores de $\mathrm{DMl}$, particularmente nos familiares de diabéticos tipo 1 com DAT.

\section{REFERÊNCIAS}

1. Jenkins RC, Weetman AP. Disease associations with autoimmune thyroid disease. Thyroid. 2002;12(11):977-88.

2. Rewers M, Norris J, Dabelea D. Epidemiology of type 1 diabetes. In: Eisenbarth GS, Lafferty K. Type 1 diabetes: molecular, cellular and clinical immunology. Chapter 9 [acesso em 2004 Nov 15]. Disponível em http://www.uchsc.edu/misc/diabetes/ eisenbook.html.

3. Bach JF. The effect of infections on susceptibility to autoimmune and allergic diseases. N Engl J Med. 2002;347:911-20.

4. Hanukoglu A, Mizrachi A, Dalal I, et al. Extrapancreatic autoimmune manifestations in type 1 diabetes and their first-degree relatives. Diabetes Care. 2003;26:1235-40.

5. Jaeger C, Petzoldt R, Hatziagelaki E, Bretzel RG. Comparative analysis of organ-specific autoantibodies in type 1 diabetic patients, their first-degree relatives, and healthy control subjects. Diabetes Care. 2001;24(1):27-32.

6. Kalicka-Kasperczyk A, Dziatkowiak H, Bartnik-Mikuta A, et al. Thyroid peroxidase antibodies and thyroid diseases in children and adolescents with newly diagnosed type 1 diabetes. Przegl Lek. 2002;59(7):509-13.

7. Onkamo P, Vaananen S, Karvonem M, et al. Worldwide increase in incidence of type 1 diabetes - the analysis of the data on published incidence trends. Diabetologia. 1999;42:1395-403.

8. Kordonouri $\mathrm{O}$, Klinghammer $\mathrm{A}$, et al. Thyroid autoimmunity in children and adolescents with type 1 diabetes. Diabetes Care. 2002;25:1346-50.
9. Ferreira SR, Franco LJ, Vivolo MA, et al. Population-based incidence of IDDM in the state of São Paulo, Brazil. Diabetes Care. 1993;16:701-4.

10. Oliveira CP, Longui CA, Monte $\mathrm{O}$, et al. Associação entre diabetes mellitus tipo 1 e doença tireoidiana auto-imune. Arq Bras Endocrinol Metab. 2004;48 Suppl 1:S547.

11. Molina LA, Fernandez CM, Perez MM, et al. Autoimmune thyroid pathology in recently diagnosed diabetes mellitus type 1. Rev Clin Esp. 1998;198(12):818-21.

12. McCanllies E, O'Leary LA, Foley TP, et al. Hashimoto's thyroiditis and insulin-dependent diabetes mellitus: differences among individuals with or without abnormal thyroid function. J Clin Endocrinol Metab. 1998;83(5):1548-51.

13. McKenzie JM, Zakarija M. Antibodies in auto-immunity thyroid disease. In: Bravaman LE, Utiger RD. Werner and Ingbar'd. The thyroid - a fundamental and clinical text. 7th ed. Philadelphia, Pennsylvania: Lippencote-Raven Publishers; 1996. p. 416-22.

14. Umpierrez GE, Latif KA, Bush A, et al. Thyroid disfunction in patients with type 1 diabetes. Diabetes Care. 2003;26:1181-5.

15. Winter WE, Harris N, Schatz D. Imunological markers in the diagnosis and prediction of autoimmune type 1a diabetes. Clin Diabetes. 2002;20(4):183-9.

16. Bosignoli R, Guimarães $A A$, Oliveira $B C$, et al. Estudo da função tireóidea em pacientes com diabetes mellitus insulinodependente (tipo 1). J Biochem Mol Biol Biophys. 1994;67(1):19-29.

17. Jorge PT, Cassaro EB. Estudo da prevalência de doença autoimune de tireóide em portadores de diabetes mellitus tipo 1. V Encontro Brasileiro de Tireóide, Foz do Iguaçu, 1993.

18. Vieira FWB. Avaliação tireoidiana da população de diabéticos tipo 1, atendida no HUAC de Campina Grande - PB, no período de janeiro de 1998 a janeiro de 1999 [monografia de conclusão do curso de especialização em análises clínicas]. Campina Grande: Universidade Estadual da Paraíba, 1999.

19. Ramos AJS, Costa ADM, Benício AVL, et al. Prevalência de doença tireoidiana em pacientes com diabetes tipo 1. Arq Bras Endocrinol Metab. 2003;47(2):177-82.

20. The Expert Committee on the Diagnosis and Classification of Diabetes Mellitus: report of the expert committee on the diagnosis and classification of diabetes mellitus. Diabetes Care. 1997;20:1183-97.

21. Grupo interdisciplinar de padronização da hemoglobina glicada A1c. A importância da hemoglobina glicada (A1c) para a avaliação do controle glicêmico em pacientes com diabetes mellitus: aspectos clínicos e laboratoriais. Posicionamento oficial - 2004 [acesso em 2005 Apr 26]. Disponível em http://diabetes.org.br/educacao/livrosonline.php.

22. Bussab WO, Morettin P. Estatística básica. $5^{\underline{a}}$ ed. São Paulo: Saraiva; 2002. p. 526.

23. Eisenbarth GS, Polonsky KS, Buse JB. Type 1 diabetes mellitus. In: Polansky KS, Melmed S, Kronenberg HM, ReedLarsen P. Williams Textbook of Endocrinology. 20. ed. Philadelphia, Pennsylvania: WB Saunders Company; 2003. p. 1485-508.

24. Karvonen M, Ptikamieni J, Tuomilehto J. The onset age of type 1 diabetes in finnish children has become younger. Diabetes Care. 1999;22(7):1066-70.

25. Molina LA, Fernandez CM, Perez MM, et al. Clinical presentation and early course of diabetes with and without thyroid autoimmunity. Diabetes Care. 1999;22(3):377-81.

26. Jorge PT, Carneiro MC, Matsumara LK, et al. Importância da determinação simultânea de anticorpos antitireoglobulina e antimicrossomal no diagnóstico de doenças auto-imunes da tireóide. Arq Bras Endocrinol Metab. 1988;32:69-71. 
27. Weetman AP, De Groot LJ. Autoimmunity to the thyroid gland. In: De Groot LJ. The thyroid and its diseases. Chapter 7 [acesso em . 2005 jan 12]. Disponível em http://www.thyroidmanager.org/thyroidbook.htm.

28. Reedlarsen P, Davies TF, Hay ID. The thyroid gland. In: Wilson JD, Foster DW, Kronenberg HM, ReedLarsen P. Williams Textbook of Endocrinology. 9th ed. Philadelphia, Pennsylvania: WB Saunders Company; 1998. p. 419-22.

29. Franzese A, Buono P, Mascolo M, et al. Thyroid autoimmunity starting during the course of type 1 diabetes denotes a subgroup of children with more severe diabetes. Diabetes Care. 2000;23(8):1201-02.

30. Hansen D, Bennedbaek FN, Hoier-Madsen M, et al. A prospective study of thyroid function, morphology and autoimmunity in young patients with type 1 diabetes. Eur $\mathrm{J}$ Endocrinol. 2003;148(2):245-51.

31. Mohn A, Di Michele S, Di Luzio R, et al. The effect of subclinical hypothyroidism on metabolic control in children and adolescents with type 1 diabetes mellitus. Diabet Med. 2002;19(1):70-3.
32. DCCT Research Group: the effect of intensive diabetes treatment on the development and progression of long-term complications in insulin-dependent diabetes mellitus: the diabetes control and complications trial. N Engl J Med. 1993;329:978-86.

33. Pousada JMDC, Britto MMS. Tratamento do diabetes melito tipo 1. In: Coronho V, Petroianu A, Santana EM, Pimenta LG. Tratado de Endocrinologia e Cirurgia Endócrina. Rio de Janeiro: Guanabara-Koogan; 2001. p. 935-51.

\section{Endereço para correspondência:}

Ricardo Rodrigues

Rua Felisberto Carrejo, 857, apto. 502 - Fundinho 38400-204 Uberlândia, MG

E-mail: drricrod@terra.com.br 